communication data rates via the space network. Missiles and smaller ELVs are often stabilized in flight by a fast (i.e. $4 \mathrm{~Hz}$ ) roll rate. This fast roll rate, combined with vehicle attitude changes, greatly increases the complexity of the high-gain antenna beam-tracking problem. Phased arrays for larger ELVs with roll control are prohibitively expensive. Prior techniques involved a traditional fully electronic phased array solution, combined with highly complex and very fast inertial measurement unit phased array beamformers.

The functional operation of this phased array is substantially different from traditional phased arrays in that it uses a hybrid electrical/mechanical beamformer that creates the relative time delays for steering the antenna beam via a small physical movement of variable delay lines. This movement is controlled via an innovative antenna control unit that accesses an internal measurement unit for vehicle attitude information, computes a beam-pointing angle to the target, then points the beam via a stepper motor controller. The stepper motor on the beamformer controls the beamformer variable delay lines that apply the appropriate time delays to the individual array elements to properly steer the beam.

The array of phased ring radiators is unique in that it provides improved gain for a small rocket or missile that uses spin stabilization for stability. The antenna pattern created is symmetric about the roll axis (like an omnidirectional wraparound), and is thus capable of providing continuous coverage that is compatible with very fast spinning rockets. For larger ELVs with roll control, a linear array of elements can be used for the 1D scanned beamformer and phased array, or a 2D scanned beamformer can be used with an $\mathrm{N} \times \mathrm{N}$ element array.

This work was done by Daniel Mullinix, Kenneth Hall, Bruce Smith, and Brian Corbin of Goddard Space Flight Center. Further information is contained in a TSP (see page 1). GSC-15805-1

\title{
(8) Mars Science Laboratory Engineering Cameras
}

\author{
NASA's Jet Propulsion Laboratory, Pasadena, California
}

NASA's Mars Science Laboratory (MSL) Rover, which launched to Mars in 2011, is equipped with a set of 12 engineering cameras. These cameras are build-to-print copies of the Mars Exploration Rover (MER) cameras, which were sent to Mars in 2003. The engineering cameras weigh $<300$ grams each and use $<3 \mathrm{~W}$ of power. Images returned from the engineering cameras are used to navigate the rover on the Martian surface, deploy the rover robotic arm, and ingest samples into the rover sample processing system. The navigation cam- eras (Navcams) are mounted to a pan/tilt mast and have a 45-degree square field of view (FOV) with a pixel scale of $0.82 \mathrm{mrad} /$ pixel.

The hazard avoidance cameras (Hazcams) are body-mounted to the rover chassis in the front and rear of the vehicle and have a 124-degree square FOV with a pixel scale of 2.1 $\mathrm{mrad} / \mathrm{pixel}$. All of the cameras utilize a frame-transfer CCD (charge-coupled device) with a $1024 \times 1024$ imaging region and red/near IR bandpass filters centered at $650 \mathrm{~nm}$. The MSL engi- neering cameras are grouped into two sets of six: one set of cameras is connected to rover computer " $\mathrm{A}$ " and the other set is connected to rover computer "B". The MSL rover carries 8 Hazcams and 4 Navcams.

This work was done by Justin N. Maki, David L. Thiessen, Ali M. Pourangi, Peter A. Kobzeff, Steven W. Lee, Arsham Dingizian, and Mark A. Schwochert of Caltech for NASA's Jet Propulsion Laboratory. For more information, contact iaoffice@jpl.nasa.gov. NPO-48550

\section{(3) Seismic Imager Space Telescope}

\section{The imager will offer alternative ways of studying earthquakes and improve early warning systems.}

\section{NASA's Jet Propulsion Laboratory, Pasadena, California}

A concept has been developed for a geostationary seismic imager (GSI), a space telescope in geostationary orbit above the Pacific coast of the Americas that would provide movies of many large earthquakes occurring in the area from Southern Chile to Southern Alaska. The GSI movies would cover a field of view as long as $300 \mathrm{~km}$, at a spatial resolution of 3 to $15 \mathrm{~m}$ and a temporal resolution of 1 to $2 \mathrm{~Hz}$, which is sufficient for accurate measurement of surface displacements and photometric changes induced by seismic waves. Computer processing of the movie images would exploit these dynamic changes to accurately measure the rapidly evolving surface waves and surface ruptures as they happen. These measurements would provide key information to advance the understanding of the mechanisms governing earthquake ruptures, and the propagation and arrest of damaging seismic waves.

GSI operational strategy is to react to earthquakes detected by ground seismometers, slewing the satellite to point at the epicenters of earthquakes above a certain magnitude. Some of these earthquakes will be foreshocks of larger earth- quakes; these will be observed, as the spacecraft would have been pointed in the right direction. This strategy was tested against the historical record for the Pacific coast of the Americas, from 1973 until the present. Based on the seismicity recorded during this time period, a GSI mission with a lifetime of 10 years could have been in position to observe at least 13 (22 on average) earthquakes of magnitude larger than 6 , and at least one (2 on average) earthquake of magnitude larger than 7 .

A GSI would provide data unprecedented in its extent and temporal and 